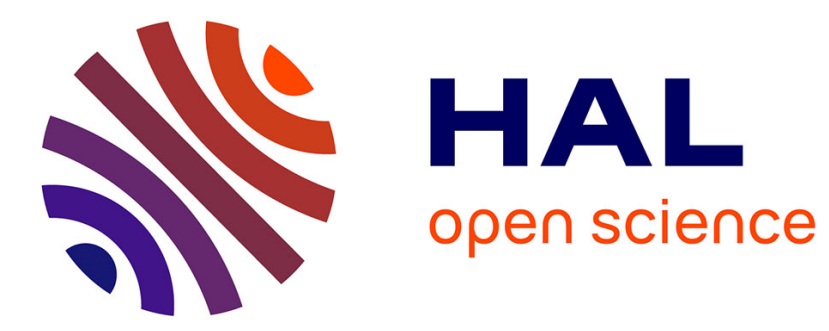

\title{
Controlling the deformation of antiferromagnetic skyrmions in the high-velocity regime
}

A Salimath, Fengjun Zhuo, R Tomasello, G Finocchio, A Manchon

\section{To cite this version:}

A Salimath, Fengjun Zhuo, R Tomasello, G Finocchio, A Manchon. Controlling the deformation of antiferromagnetic skyrmions in the high-velocity regime. Physical Review B, 2020, 101, 10.1103/physrevb.101.024429 . hal-03031103

\section{HAL Id: hal-03031103 https://hal.science/hal-03031103}

Submitted on 30 Nov 2020

HAL is a multi-disciplinary open access archive for the deposit and dissemination of scientific research documents, whether they are published or not. The documents may come from teaching and research institutions in France or abroad, or from public or private research centers.
L'archive ouverte pluridisciplinaire HAL, est destinée au dépôt et à la diffusion de documents scientifiques de niveau recherche, publiés ou non, émanant des établissements d'enseignement et de recherche français ou étrangers, des laboratoires publics ou privés. 


\title{
Controlling the deformation of antiferromagnetic skyrmions in the high-velocity regime
}

\author{
A. Salimath, ${ }^{1, *}$ Fengjun Zhuo $\odot,{ }^{1}$ R. Tomasello $\odot,{ }^{2}$ G. Finocchio, ${ }^{3}$ and A. Manchon $\oplus^{1, \dagger}$ \\ ${ }^{1}$ King Abdullah University of Science and Technology (KAUST), Physical Science and Engineering Division (PSE), \\ Thuwal 23955-6900, Saudi Arabia \\ ${ }^{2}$ Institute of Applied and Computational Mathematics, FORTH, GR-70013 Heraklion-Crete, Greece \\ ${ }^{3}$ Department of Mathematical and Computer Sciences, Physical Sciences, and Earth Sciences, University of Messina, I-98166 Messina, Italy
}

(Received 24 July 2019; revised manuscript received 5 December 2019; published 31 January 2020)

\begin{abstract}
While antiferromagnetic skyrmions display appealing properties, their lateral expansion in the high-velocity regime hinders their potential for applications. In this work, we study the impact of spin Hall torque, spin transfer torque, and topological torque on the velocity-current relation of antiferromagnetic skyrmions with the aim of reducing this deformation. Using a combination of micromagnetic simulations and analytical derivations, we demonstrate that the lateral expansion of the antiferromagnetic skyrmion is reminiscent of the well-known Lorentz contraction identified in one-dimensional antiferromagnetic domain walls. We also show that in the flow regime the lateral expansion is accompanied by a progressive saturation of the skyrmion velocity when driven by spin Hall and topological torques. This saturation occurs at much smaller velocities when driven by the topological torque, while the lateral expansion is reduced, preventing the skyrmion size from diverging at large current densities. We extend this study toward synthetic antiferromagnets, where the weaker antiferromagnetic exchange leads to much larger lateral expansion at smaller current densities in all cases. This study suggests that a compromise must be made between skyrmion velocity and lateral expansion during the device design. In this respect, exploiting the topological torque could lead to better control of the skyrmion velocity in antiferromagnetic racetracks.
\end{abstract}

DOI: 10.1103/PhysRevB.101.024429

\section{INTRODUCTION}

For decades, ferromagnets have been the preferred active layer for spintronic devices, maintaining antiferromagnets to a passive role in spite of their superior properties. Indeed, antiferromagnets are abundant in nature, they are robust against reasonably large $(\sim$ a few teslas) external magnetic fields, and they display very high frequency dynamics in the terahertz range [1]. The recent prediction [2,3] and observation of current-driven manipulation of the Néel order of CuMnAs [4], $\mathrm{Mn}_{2} \mathrm{Au}$ [5], $\mathrm{NiO}$ [5,6], and synthetic antiferromagnets [7] open thrilling perspectives for data storage and memory applications $[8,9]$. Antiferromagnets are particularly appealing in the context of the racetrack memory [10], where information is stored in magnetic domain walls and controlled electrically via spin transfer [11,12] or spin-orbit torque [13]. As a matter of fact, theoretical predictions have suggested that antiferromagnetic domain walls could be immune to Walker breakdown and reach velocities as high as tens of kilometers per second [14-17]. In this context, the recent prediction of antiferromagnetic skyrmions bears inspiring promise for futuristic ultrahigh-density storage $[18,19]$.

Magnetic skyrmions are a class of topologically protected objects observed in chiral magnets [20-24], i.e., bulk materials or heterostructures lacking inversion symmetry. Recent experiments have reported skyrmion sizes down to a few

\footnotetext{
*akshaykumar.salimath@kaust.edu.sa

†aurelien.manchon@kaust.edu.sa
}

nanometers in transition metal ferromagnets [25-28], which is crucial for high-density data storage. Their rich topological properties, such as room-temperature stability, current-driven motion, and reduced interaction with local disorder, have been well established both experimentally and theoretically [26,2934]. In addition, their topological signatures make skyrmion detection in ferromagnets rather simple [35]. Besides these attractive features, ferromagnetic skyrmions also experience current-driven gyroscopic forces that can limit their scalability $[31,32,36]$. On the other hand, it was recently suggested that such gyroscopic forces could be exploited to enhance the mobility of a train of skyrmions in ferromagnetic nanotracks [37].

In contrast, the progress in antiferromagnetic skyrmions has been limited. The absence of gyroscopic forces combined with vanishing stray field make antiferromagnets promising materials for spintronic circuits [38-41]. The most recent theoretical works unambiguously suggest higher skyrmion mobility in antiferromagnets [41,42]. In addition, the nontrivial topology of antiferromagnetic skyrmions enables the flow of topological spin Hall currents [43,44]. These local spin currents lead to a topological torque that can significantly enhance their mobility compared to their ferromagnetic counterpart [45]. In spite of these appealing properties, recent numerical simulations have pointed out that antiferromagnetic skyrmions have the tendency to exhibit lateral deformation when driven at high velocity [46], which can severely hinder their potential for a range of applications. As a matter of fact, this expansion can lead to skyrmion annihilation when the lateral size becomes comparable to the width of the antiferromagnetic track. Hence, controlling the lateral expansion 
(a)

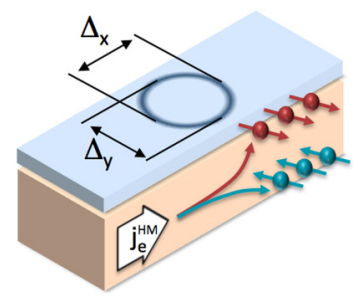

(b)

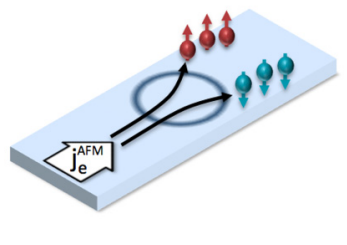

FIG. 1. Schematics of the current-driven motion of an antiferromagnetic skyrmion upon (a) spin Hall torque arising from an adjacent metal and (b) topological torque arising from electron flowing along the magnetic texture. $j_{e}^{\mathrm{HM}}$ and $j_{e}^{\mathrm{AFM}}$ denote the current density flowing in the metallic layer and in the antiferromagnetic layer, respectively. The antiferromagnetic skyrmion is represented by the dark blue circle and its longitudinal and lateral widths are defined in (a). The curved red and blue arrows denote the bent trajectory of up and down spins upon the spin Hall effect.

of antiferromagnetic skyrmions is crucial for enabling their integration in devices.

In the present work, we investigate the impact of various forms of spin torque on the velocity-current relation of antiferromagnetic skyrmions with the aim of reducing their lateral deformation in the high-velocity regime. Focusing on collinear antiferromagnets, we consider two main scenarios, depicted in Fig. 1: in the first scenario, the skyrmion is driven by the spin-orbit torque due to the spin current generated in an adjacent heavy metal [13]; in the second scenario, the current flows in the magnetic layer itself, generating the adiabatic, nonadiabatic, and topological torques [45]. This article is organized as follows. In Sec. II A, using analytical derivations based on the Lagrangian formalism, we show that the lateral expansion of the antiferromagnetic skyrmion is reminiscent of the well-known Lorentz contraction reported in one-dimensional antiferromagnetic domain walls [16,17,4749]. In Sec. II B, using micromagnetic simulations, we report that the skyrmion displays very different dynamical properties when driven by the spin-orbit torque and when driven by the topological torque. In the former, the linear current-velocity relation is accompanied by a divergence of the lateral expansion close to the maximum velocity, set by the spin wave velocity. In the latter, the current-velocity relation is sublinear for the same range of current density, a property ascribed to the peculiar structure of the topological torque, leading to a much smaller lateral expansion. This observation suggests that the topological torque could be exploited to better control the skyrmion velocity and deformation when designing the antiferromagnetic racetrack. In Sec. III, we extend this study to synthetic antiferromagnets and show that the very weak antiferromagnetic exchange results in much smaller velocities and much larger deformation at reasonable current densities. The conclusion is given in Sec. IV.

\section{DYNAMICS OF ANTIFERROMAGNETIC SKYRMIONS}

\section{A. Lagrangian formalism for skyrmion motion}

To guide the analysis of the numerical simulations and establish some physical insights, we first derive the equa- tion of motion of an antiferromagnetic skyrmion based on the Lagrangian formalism [14,49,50]. As explained in the introduction, we consider the two main scenarios depicted in Fig. 1: (i) either the skyrmion is driven by the spin Hall torque arising from an adjacent heavy metal [Fig. 1(a)], or the skyrmion is driven by the regular spin transfer torque induced by electrons flowing along the magnetic texture [Fig. 1(b)]. In the latter case, the nontrivial topology of the magnetic skyrmion promotes the emergence of the topological spin Hall effect $[43,44]$, which induces a so-called "topological torque" that enhances the nonadiabaticity [45]. We stress that similarly to the topological spin Hall effect, the topological torque produces a global force on the magnetic texture only when this texture presents a nontrivial topology.

Considering the nonlinear sigma model of a bipartite collinear antiferromagnet in the continuum approximation [51], the total magnetization field and antiferromagnetic order parameter are defined $\mathbf{m}(\mathbf{r}, t) \equiv \mathbf{m}_{1}(\mathbf{r}, \mathbf{t})+\mathbf{m}_{2}(\mathbf{r}, \mathbf{t})$ and $\mathbf{l}(\mathbf{r}, \mathbf{t}) \equiv \mathbf{m}_{\mathbf{1}}(\mathbf{r}, \mathbf{t})-\mathbf{m}_{\mathbf{2}}(\mathbf{r}, \mathbf{t})$, where $\mathbf{m}_{1}$ and $\mathbf{m}_{2}$ are the magnetizations on adjacent sublattices, respectively. The normalized staggered vector field is defined $\mathbf{n}(\mathbf{r}, \mathbf{t}) \equiv \mathbf{I}(\mathbf{r}, \mathbf{t}) /|\mathbf{I}(\mathbf{r}, \mathbf{t})|$. The skyrmion motion is described by its central position $\mathbf{r}_{c}$, longitudinal width $\Delta_{x}$, and lateral width $\Delta_{y}$. In the continuum approximation, the Lagrangian for the antiferromagnet system is written as [52]

$$
\begin{aligned}
\mathcal{L}[\mathbf{n}]= & \int d V\left\{\frac{\rho}{2}|\dot{\mathbf{n}}|^{2}-\frac{A_{\mathrm{AFM}}}{2} \sum_{i}\left|\partial_{i} \mathbf{n}\right|^{2}+\frac{K_{z}}{2}(\mathbf{n} \cdot \hat{\mathbf{z}})^{2}\right. \\
& \left.-\frac{D}{2} \mathbf{n} \cdot[(\hat{\mathbf{z}} \times \nabla) \times \mathbf{n}]\right\},
\end{aligned}
$$

where the overdot $\left\langle{ }^{\circ}\right\rangle$ denotes the time derivative, while $\partial_{i}$ denotes the spatial derivative taken along the direction $\hat{\mathbf{i}}$. In addition, $\rho=\left(M_{\mathrm{s}} / \gamma\right)^{2} \chi$ quantifies the inertia of the staggered magnetization, where $\chi>0$ is proportional to magnetic susceptibility. $M_{\mathrm{S}}$ is saturation magnetization on one sublattice, $\gamma$ is the absolute value of the gyromagnetic ratio, $A_{\mathrm{AFM}}$ is the antiferromagnetic exchange constant, $D$ is the DzyaloshinskiiMoriya interaction (DMI), and $K_{z}$ is the uniaxial anisotropy constant. In Eq. (1), the nonequilibrium magnetization $\mathbf{m}(\mathbf{r}, t)$ does not appear explicitly as it is a slave variable $[14,15]$. This assumption is only valid for smooth and slow dynamics in the presence of large antiferromagnetic exchange. The Rayleigh dissipation functional of an antiferromagnet accounting for the Gilbert damping and the dissipative torques reads

$$
\begin{aligned}
\mathcal{R}_{0}= & \alpha \frac{M_{\mathrm{s}}}{\gamma} \int d V\left\{\left(\partial_{t}+\frac{\beta}{\alpha} b_{j} \partial_{x}\right) \mathbf{n}\right. \\
& \left.+\frac{\lambda^{2}}{\alpha} b_{j} \mathcal{N}_{x y}\left(\partial_{y} \mathbf{n} \times \mathbf{n}\right)+\frac{\tau_{\mathrm{sh}}}{\alpha}(\hat{\mathbf{y}} \times \mathbf{n})\right\}^{2},
\end{aligned}
$$

where $b_{j}=\mu_{B} j_{e}^{\mathrm{AFM}} P / e M_{\mathrm{s}}$ is the magnitude of the adiabatic torque, $P$ is the spin polarization, $j_{e}^{\mathrm{AFM}}$ is the current density flowing in the antiferromagnetic layer [Fig. 1(b)], $\alpha$ is the Gilbert damping constant, and $\beta$ is the nonadiabaticity associated with spin relaxation [53]. In Appendix A, we propose a phenomenological derivation of the nonadiabaticity in collinear antiferromagnets. The second term is a damping term induced by the intrinsic topological nonadiabatic 
spin torque of an antiferromagnetic skyrmion [45], where $\mathcal{N}_{x y}=\left(\partial_{x} \mathbf{n} \times \partial_{y} \mathbf{n}\right) \cdot \mathbf{n}$ is the topological charge density for the staggered field $\mathbf{n}$ and $\lambda$ is the phenomenological parameter describing the topological torque magnitude. The third term is the contribution from the spin-orbit torque due to spin Hall effect. It reads [13] $\tau_{\mathrm{sh}}=\left(\gamma / M_{\mathrm{s}} t\right)(\hbar / 2 e) \xi \theta_{\mathrm{sh}} j_{e}^{\mathrm{HM}}$, where $\theta_{\mathrm{sh}}$ is the spin Hall angle, $\xi$ is the spin transparency, $t$ is the thickness of the magnetic layer, and $j_{e}^{\mathrm{HM}}$ is the current density flowing in the adjacent metallic layer, as depicted in Fig. 1(a). These three torques are all even in $\mathbf{n}$ and proportional to the injected current. Upon time reversal, these torques change sign, revealing their dissipative nature.

It is convenient to consider the staggered vector $\mathbf{n}$ in spherical coordinates, $\mathbf{n}=(\sin \theta \cos \varphi, \sin \theta \sin \varphi, \cos \theta)$, where the polar angle $\theta$ and azimuthal angle $\varphi$ are given by

$$
\begin{gathered}
\theta=4 \tan ^{-1}\left[\exp \left(\sqrt{X^{2}+Y^{2}}\right)\right], \\
\varphi=m \tan ^{-1}\left(\frac{Y}{X}\right)+\zeta .
\end{gathered}
$$

Here, $m$ and $\zeta$ are the skyrmion vorticity and helicity [18], respectively, $X=\frac{x-r(t)}{\Delta_{x}(t)}$, and $Y=\frac{y}{\Delta_{y}(t)}$, where $r(t)$ is the skyrmion position. Without loss of generality, we set $m=1$ and $\zeta=0$. The antiferromagnetic skyrmion travels in a perfectly straight trajectory along the current because of the countervailing opposite winding numbers on two sublattices [41]. The equation of motion of the antiferromagnetic skyrmion is obtained by solving the Euler-Lagrange equation

$$
\frac{\delta \mathcal{L}}{\delta \eta}-\frac{d}{d t} \frac{\delta \mathcal{L}}{\delta \dot{\eta}}+\frac{\delta \mathcal{R}}{\delta \dot{\eta}}=0
$$

where $\eta$ stands for $r, \Delta_{x}$, and $\Delta_{y}$. We emphasize that in magnetic domain walls the DMI also induces a distortion of the azimuthal angle, as pointed out in Ref. [54]. This spatially dependent tilting, conjugated to the domain wall width, scales with the ratio between the DMI and magnetic exchange and is therefore neglected in the present work. The good match between the analytical and numerical results validates this assumption a posteriori. In the following, we adopt the rigidmotion assumption, assuming that the skyrmion velocity is constant $(\dot{r}=v$ and $\ddot{r}=0)$ and its deformation does not change over time $\left(\dot{\Delta}_{x, y}=0\right)$. After straightforward but rather tedious algebra, we obtain the following coupled equations:

$$
\begin{gathered}
v=-\left(\beta+C_{1} \frac{\lambda^{2}}{\Delta_{y}^{2}}\right) \frac{b_{j}}{\alpha}-C_{2} \frac{\tau_{\mathrm{sh}} \Delta_{x}}{\alpha}, \\
C_{3} \frac{\pi K_{z}}{D}=\frac{1}{\Delta_{x}}+\frac{1}{\Delta_{y}}, \\
2 \rho \frac{v^{2}}{\Delta_{x}^{2}}=-2 A_{\mathrm{AFM}}\left(\frac{1}{\Delta_{y}^{2}}-\frac{1}{\Delta_{x}^{2}}\right)+C_{2} D\left(\frac{1}{\Delta_{x}}-\frac{1}{\Delta_{y}}\right) .
\end{gathered}
$$

The coefficients $C_{1,2,3}$ are defined

$$
C_{1}=\frac{I_{3}}{I_{1}+I_{2}}, C_{2}=-\frac{I_{4}+I_{5}}{I_{1}+I_{2}}, C_{3}=-\frac{1}{I_{4}+I_{5}},
$$

and

$$
\begin{gathered}
I_{1}=\iint_{-\infty}^{+\infty} d X d Y\left(\frac{2 X}{R \cosh R}\right)^{2}, \\
I_{2}=\iint_{-\infty}^{+\infty} d X d Y \sin ^{2} \theta\left(\frac{Y}{R^{2}}\right)^{2}, \\
I_{3}=\iint_{-\infty}^{+\infty} d X d Y \sin ^{2} \theta\left(\frac{2}{R \cosh R}\right)^{2}, \\
I_{4}=\iint_{-\infty}^{+\infty} d X d Y \frac{2 X^{2}}{R^{2} \cosh R}, \\
I_{5}=\iint_{-\infty}^{+\infty} d X d Y \sin \theta \cos \theta \frac{Y^{2}}{R^{3}},
\end{gathered}
$$

where $R=\sqrt{X^{2}+Y^{2}}$. Numerically, we obtain $C_{1}=2.53$, $C_{2}=0.09$, and $C_{3}=0.6$. Equations (6)-(8) constitute the basis of the analysis provided in the following sections. Solving Eqs. (7) and (8) provides the explicit expression of the longitudinal and lateral widths of the skyrmion as a function of the velocity. We obtain

$$
\begin{gathered}
\Delta_{x}=\frac{\Delta_{0}}{2}\left[1+\sqrt{1-\left(v / v_{m}\right)^{2}}\right], \\
\Delta_{y}=\frac{\Delta_{0}}{2}\left[1+\frac{1}{\sqrt{1-\left(v / v_{m}\right)^{2}}}\right],
\end{gathered}
$$

where $\Delta_{0}=2 D / \pi C_{3} K_{z}$ is the width at zero velocity and $v_{m}=\sqrt{\left(A_{\mathrm{AFM}}+\Delta_{0} C_{2} D / 4\right) / \rho}$ is the spin wave velocity. We emphasize that these two expressions are completely independent of the nature of the torque considered. In other words, the skyrmion deformation only depends on its velocity and the lateral expansion diverges when the skyrmion velocity reaches the spin wave velocity. These relations are a reminiscence of the Lorentz contraction identified for a one-dimensional antiferromagnetic domain wall [16,17,47-49]. The divergence of the lateral expansion of the skyrmion has been observed numerically [46] and poses a daring challenge for the design of antiferromagnetic racetracks. As a matter of fact, although the maximum velocity of antiferromagnetic skyrmions is in principle very high (a few kilometers per second [16,17]), their lateral deformation can lead to skyrmion annihilation when the skyrmion edges reach the boundaries of the track [46].

\section{B. Micromagnetic simulations}

We now move on to the numerical simulations. In the micromagnetic framework, the skyrmion motion within each sublattice $i$ is governed by the Landau-Lifshitz-Gilbert equation [55],

$$
\begin{aligned}
\dot{\mathbf{m}}_{i}=- & \gamma \mu_{0} \mathbf{m}_{i} \times \mathbf{H}_{\text {eff }}^{i}+\alpha \mathbf{m}_{i} \times \dot{\mathbf{m}}_{i}+\tau_{\mathrm{sh}} \mathbf{m}_{i} \times\left(\mathbf{y} \times \mathbf{m}_{i}\right) \\
& -b_{j} \partial_{x} \mathbf{m}_{i}+\beta b_{j} \mathbf{m}_{i} \times \partial_{x} \mathbf{m}_{i}+b_{j} \lambda^{2} \mathcal{N}_{x y} \partial_{y} \mathbf{m}_{i},
\end{aligned}
$$

where $\mu_{0} \mathbf{H}_{\text {eff }}^{i}=-\left(1 / M_{\mathrm{s}}\right) \delta W / \delta \mathbf{m}_{i}$ is the effective field, $W$ being the magnetic energy density and $M_{\mathrm{S}}$ the saturation magnetization. The effective field includes the contributions from the exchange fields, the perpendicular uniaxial anisotropy $K_{z}$, the interfacial DMI $D$, and the demagnetizing field. The exchange fields take into account both ferromagnetic coupling 
between neighbors in each sublattice (this is the same as in the standard model for the ferromagnets) and the antiferromagnetic coupling between the two sublattices [56,57],

$$
\begin{aligned}
& \mathbf{h}_{\mathrm{ex}, 1}=a_{\mathrm{FM}} \nabla^{2} \mathbf{m}_{1}+\lambda_{\mathrm{AFM}} \mathbf{m}_{2}, \\
& \mathbf{h}_{\mathrm{ex}, 2}=a_{\mathrm{FM}} \nabla^{2} \mathbf{m}_{2}+\lambda_{\mathrm{AFM}} \mathbf{m}_{1},
\end{aligned}
$$

where $a_{\mathrm{FM}}=2 A_{\mathrm{FM}} / \mu_{0} M_{\mathrm{s}}^{2}$ and $\lambda_{\mathrm{AFM}}=4 A_{\mathrm{AFM}} / \mu_{0} a^{2} M_{\mathrm{s}}^{2}$, with $A_{\mathrm{FM}}(>0)$ and $A_{\mathrm{AFM}}(<0)$ being the intra-sublattice ferromagnetic and inter-sublattice antiferromagnetic exchange constants, respectively; $a$ is the lattice constant and $\mu_{0}$ is the permeability of vacuum. The third term in Eq. (16) corresponds to the spin Hall torque $\tau_{\mathrm{sh}}$ [Fig. 1(a)], and the fourth, fifth, and sixth torque terms are the contributions from the adiabatic torque $b_{j}$, the nonadiabatic torque $\beta b_{j}$, and the topological torque $\lambda^{2} b_{j}$ [45] [Fig. 1(b)]. Electrons flow toward the right for $j_{e}>0$. In the simulations, we consider a collinear antiferromagnet with each sublattice size $1000 \times 100 \times 1 \mathrm{~nm}^{3}$. The layer is discretized into cells of volume $2 \times 2 \times 1 \mathrm{~nm}^{3}$. The material parameters are $M_{s}=376 \mathrm{kA} / \mathrm{m}, A_{\mathrm{FM}}=-A_{\mathrm{AFM}}=$ $6.59 \mathrm{pJ} / \mathrm{m}, K_{z}=0.15 \mathrm{MJ} / \mathrm{m}^{3}, D=1.0 \mathrm{MJ} / \mathrm{m}^{2}$, and $\alpha=$ 0.005 . For these parameters, a relaxed skyrmion size of $20 \mathrm{~nm}$ is obtained.

\section{Skyrmion motion under spin Hall torque}

First we consider the case of skyrmion motion under spin Hall torque. To do so, we set $\theta_{\mathrm{sh}}=0.12$, and turn off the three torques arising from current flowing in the bulk of the antiferromagnet, $b_{j}=0$. Then, Eqs. (6)-(8) give

$$
v=-v_{\mathrm{sh}} \frac{\Delta_{x}}{\Delta_{0}}=-\frac{v_{\mathrm{sh}}}{2}\left[1+\sqrt{1-\left(v / v_{m}\right)^{2}}\right],
$$

where $v_{\mathrm{sh}}=\tau_{\mathrm{sh}} C_{2} \frac{\Delta_{0}}{\alpha}$. We emphasize that for reasonable parameters, $v_{\mathrm{sh}} \ll v_{m}$, so that the velocity is expected to remain linear as a function of the current density. Figure 2(a) shows snapshots of the simulated skyrmion motion at different current densities. The antiferromagnetic skyrmion undergoes a lateral expansion at high current densities, as predicted by our model [see Eq. (15)]. The corresponding skyrmion velocity and its lateral and longitudinal expansions are reported in Figs. 2(b) and 2(c), respectively. The solid lines represent the numerical simulations, while the dotted lines are the analytical predictions from Eq. (19). At reasonable applied currents, the skyrmion velocity can reach a few kilometers per second, which is significantly larger than the velocities observed in ferromagnets. The skyrmion velocity increases linearly at low current densities, followed by a slight nonlinear behavior at high current densities, not reproduced by our analytical theory. This nonlinearity is associated with the skyrmion lateral expansion in the high-velocity regime, as reported on Fig. 2(c). A possible source for the discrepancy between the numerical and analytical results is the deformation of the azimuthal angle pointed out by Kravchuk [54] and neglected in our analytical model. Notice that the saturation exposed in Eq. (19) is never reached in our simulations because the boundaries of the magnetic wire prevent further lateral expansion of the skyrmion, leading to its eventual destruction at very large current densities. The strong gyroscopic forces on individual skyrmions in each sublattice and the strong

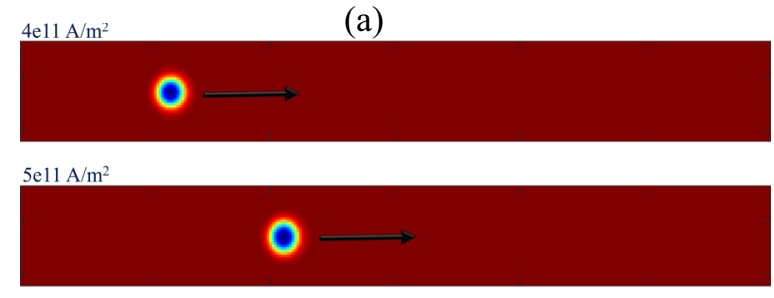

$6 \mathrm{e} 11 \mathrm{~A} / \mathrm{m}^{2}$

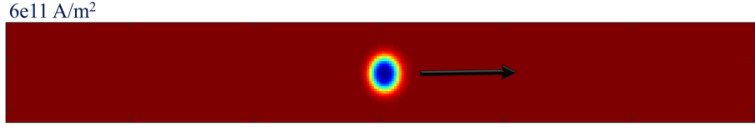

$7 \mathrm{e} 11 \mathrm{~A} / \mathrm{m}^{2}$
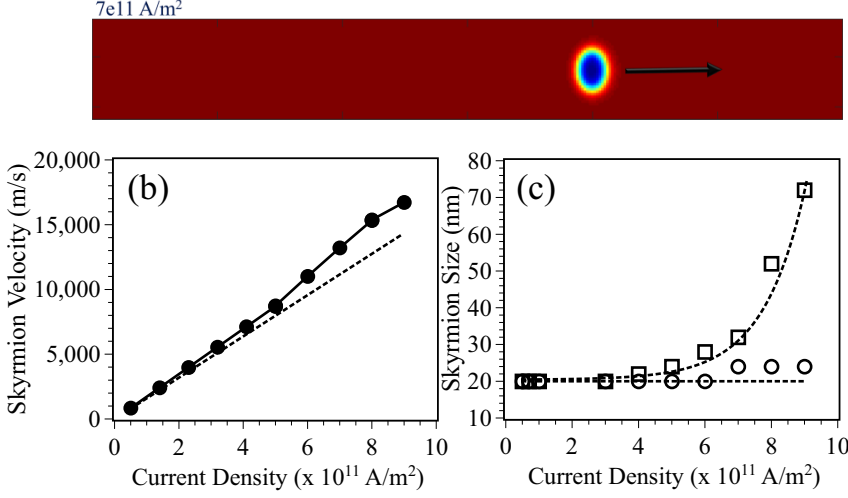

FIG. 2. Skyrmion motion in an antiferromagnet under spin Hall torque. (a) Simulated snapshots of spin Hall torque driven skyrmion motion showing the skyrmion deformation at high current densities. (b) Simulated skyrmion velocity-current characteristics (black dots). The dashed line shows the fit using Eq. (19). (c) Simulated skyrmion size variation, $\Delta_{x}$ (open circles) and $\Delta_{y}$ (open squares), as a function of applied current density. The dashed lines correspond to Eqs. (14) and (15). The scattered values of $\Delta_{x, y}$ are due to the difficulty in accurately extracting the skyrmion size in the simulations due to the large micromagnetic unit cell $(2 \mathrm{~nm})$.

antiferromagnetic coupling between the sublattice result in a pronounced expansion along the lateral direction $\Delta_{y}$, while the longitudinal width $\Delta_{x}$ remains mostly constant [Fig. 2(c)]. Overall, the fits using Eqs. (15) and (14) are in reasonable agreement with the simulation, demonstrating that the lateral expansion of antiferromagnetic skyrmions is an inherent characteristic.

\section{Skyrmion motion under spin transfer torque}

Next, we focus on the influence of spin transfer torque on the skyrmion motion [Fig. 1(b)]. As explained above, the nontrivial topology of antiferromagnetic skyrmions induces a topological spin Hall effect that contributes to an additional topological torque. This effect was identified experimentally in magnetic vortices [58] and is expected to substantially influence the dynamics of skyrmions [37,59], especially upon reducing their size. Let us first neglect this topological contribution and only consider the conventional spin transfer torque. Based on Eqs. (6)-(8), the velocity reads

$$
v=-\frac{\beta b_{j}}{\alpha} .
$$

In other words, the velocity is independent of the skyrmion size and is expected to be linear as a function of the injected 

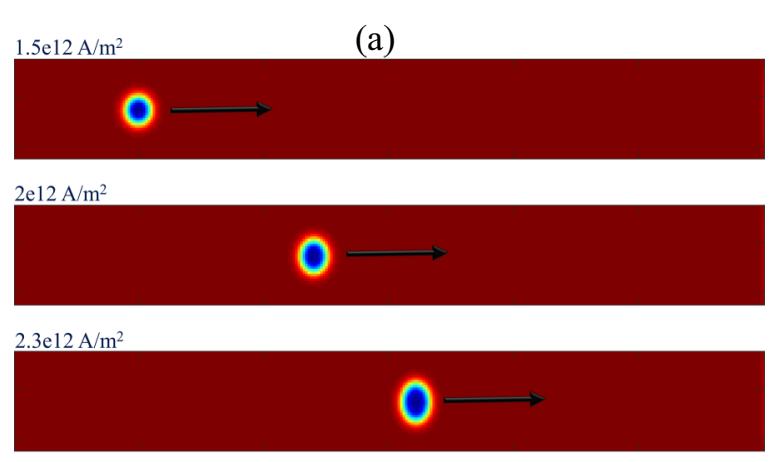

$2.5 \mathrm{e} 12 \mathrm{~A} / \mathrm{m}^{2}$
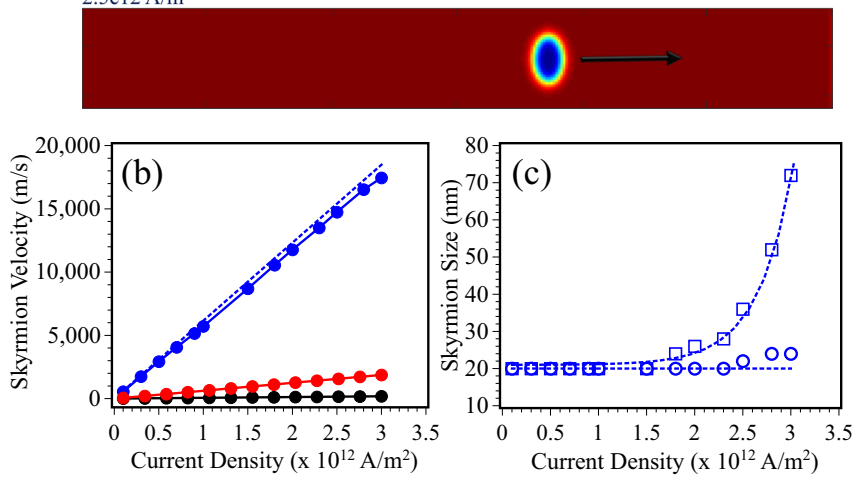

FIG. 3. Skyrmion motion in an antiferromagnet under spin transfer torque. (a) Snapshots of spin transfer torque driven skyrmion motion showing the texture deformation at high current densities for $\beta=100 \alpha$. (b) Simulated skyrmion velocity-current characteristics for $\beta=\alpha$ (black), $\beta=10 \alpha$ (red), and $\beta=100 \alpha$ (blue). The dashed line is a fit using Eq. (20). (c) Simulated skyrmion size variation, $\Delta_{x}$ (open circles) and $\Delta_{y}$ (open squares), as a function of applied current density for $\beta=100 \alpha$. The dashed lines correspond to Eqs. (14) and (15).

current density. Notice that this linearity is only valid as long as the skyrmion is rigid; i.e., the only deformation allowed is a change in the skyrmion size through $\Delta_{x}$ and $\Delta_{y}$. Under this assumption, the velocity is linear even when $v \rightarrow v_{m}$, whereas in the spin Hall case discussed above, a nonlinearity occurs in this case. This distinct behavior is ascribed to the very structure of the torques: whereas the spin transfer torque is proportional to the longitudinal gradient $\sim \partial_{x} \mathbf{m}_{i}$, the spin Hall torque is independent of it. Still, in both cases, the lateral expansion occurs around $v \approx v_{m}$, as expected from the discussion of Sec. II A.

Figure 3(a) shows snapshots of the simulated skyrmion motion at different current densities, while the dependence of the velocity as a function of the applied current density is reported in Fig. 3(b). In experiments, $\beta$ is on the order of $\alpha$ [60-62], which results in very small skyrmion velocities (solid black line). In order to obtain velocities comparable to that induced by spin Hall torque [see Fig. 2(b)], we need to consider unrealistically large values of the nonadiabaticity parameter $(\beta=10 \alpha$ and $\beta=$ $100 \alpha)$. Whereas increasing the nonadiabaticity parameter does not modify the linearity of the velocity, it triggers the skyrmion lateral expansion as expected in such a high-velocity regime.
Let us now turn on the topological torque. Solving Eqs. (6)-(8) for this torque only, we obtain

$$
v=-v_{\text {top }}\left(\frac{\Delta_{0}}{\Delta_{y}}\right)^{2}=-4 v_{\text {top }} \frac{1-\left(v / v_{m}\right)^{2}}{\left[1+\sqrt{1-\left(v / v_{m}\right)^{2}}\right]^{2}},
$$

where $v_{\text {top }}=C_{1} \frac{\lambda^{2}}{\Delta_{0}^{2}} \frac{b_{j}}{\alpha}$. In the limit of small velocity, $v \ll v_{m}$, we obtain $v \approx-v_{\text {top }}$, while close to the spin wave velocity, $v \sim v_{m}$, we obtain

$$
v=\frac{v_{\text {top }} v_{m}}{v_{\text {top }}-v_{m} / 8}
$$

In other words, we expect that the saturation of the skyrmion velocity should happen at smaller current densities, corresponding to $v_{\text {top }} \sim v_{m} / 8$, rather than $v_{\text {sh }} \sim v_{m}$ for spin Hall torque [see Eq. (19)]. We emphasize that $v_{\text {top }} \propto 1 / \Delta_{0}^{2}$, which means that it increases significantly upon reducing the skyrmion size. In addition, previous numerical estimates suggest that the parameter $\lambda$, which is associated with spin precession about the emergent magnetic field, is about 1 to $3 \mathrm{~nm}$ [45]. Therefore, in the scenario depicted in Fig. 1, the topological torque is likely to dominate over the nonadiabatic torque for a skyrmion size of $20 \mathrm{~nm}$ or less.

Figure 4(a) shows snapshots of the simulated skyrmion motion at different current densities when both nonadiabatic and topological torques are present. In the high-velocity regime, we see that the skyrmion texture remains mostly unchanged, in spite of the high velocity of the skyrmion. In Fig. 4(b), we plot skyrmion velocity as a function of applied current density for several values of topological torque parameter $\lambda^{2}$, keeping $\beta=\alpha$ for all the simulations. With this set of parameters, we see that the skyrmion velocity can reach up to $14 \mathrm{~km} / \mathrm{s}$, which is comparable to the velocity obtained for the spin Hall torque [Fig. 2(b)]. In the case of conventional spin transfer torque, such a velocity can only be obtained by setting an unrealistically high $\beta$ parameter [Fig. 3(b)]. Hence, the topological torque can induce a very high skyrmion velocity at reasonable current densities. Interestingly, in the high-velocity regime, we observe a saturation of the velocity that occurs at much lower velocities compared to the spin Hall torque case. We notice that the maximum velocity remains the same as in the spin Hall torque case, as it is given by the spin wave velocity $v_{m}$ in both cases.

We can understand this distinct behavior by looking at Eq. (6). The velocity contribution due to the topological torque, $v \propto \lambda^{2} / \Delta_{y}^{2}$, is much more sensitive to the change in width than the contribution due to the spin Hall torque, $v \propto \tau_{\text {sh }} \Delta_{x}$. Therefore, whereas $\Delta_{x}$ remains mostly constant, the increase in lateral width $\Delta_{y}$ progressively quenches the influence of the topological torque as the skyrmion enters the high-velocity regime, thereby resulting in the sublinear dependence observed in Fig. 4(b). We emphasize that even a moderate modification of $\Delta_{y}$, as reported on Fig. 4(c), is sufficient to dramatically reduce the influence of the topological torque. In contrast, when driven by the spin Hall torque, the velocity contribution is proportional to $\Delta_{x}$, which remains mostly constant in the high-velocity regime. In this latter case, nonlinearities only appear at higher current densities, close to the maximum velocity. The sublinear current-velocity relation that occurs in the case of topological torque results in 
(a)
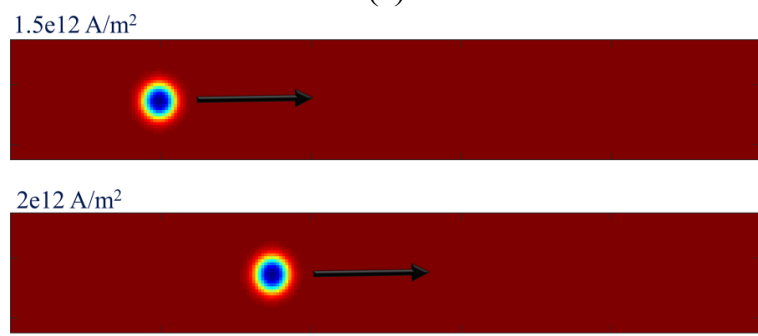

$2.3 \mathrm{e} 12 \mathrm{~A} / \mathrm{m}^{2}$

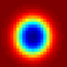

$2.5 \mathrm{e} 12 \mathrm{~A} / \mathrm{m}^{2}$
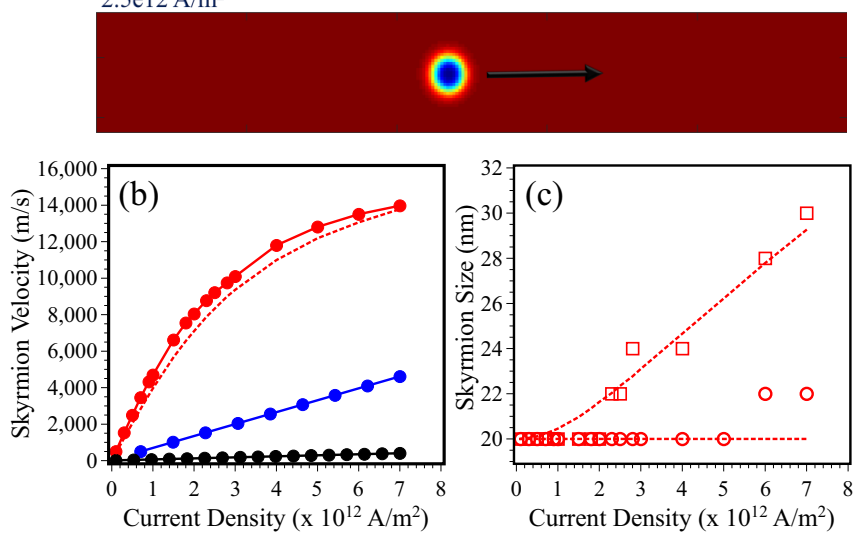

FIG. 4. Skyrmion motion in an antiferromagnet under topological torque. (a) Simulated snapshots of topological torque driven skyrmion motion at high current densities. (b) Simulated skyrmion velocity-current characteristics for $\beta=\alpha$ and $\lambda^{2}=0$ (black), $\lambda^{2}=$ $1.6 \mathrm{~nm}^{2}$ (blue), and $\lambda^{2}=13 \mathrm{~nm}^{2}$ (red). The dashed line is a fit using Eq. (21). (c) Simulated skyrmion size variation, $\Delta_{x}$ (open circles) and $\Delta_{y}$ (open squares), as a function of applied current density for $\beta=\alpha$ and $\lambda^{2}=13 \mathrm{~nm}^{2}$. The dashed lines correspond to Eqs. (14) and (15). The small variation of the skyrmion shape and the relatively large size of the micromagnetic unit cell $(2 \mathrm{~nm})$ make the accurate estimation of $\Delta_{x, y}$ difficult.

a "self-control" of the skyrmion lateral expansion, as shown in Fig. 4(c). Since the skyrmion experiences a slowdown, its expansion remains limited. This property is particularly interesting for the design of antiferromagnetic racetracks. The moderate lateral expansion resulting from the progressive quenching of the topological torque at high velocities ensures the motion of the skyrmion at relatively high velocity without risking its annihilation.

\section{SKYRMION MOTION IN SYNTHETIC ANTIFERROMAGNETS}

We conclude this study by considering the case of synthetic antiferromagnets. Synthetic antiferromagnets are easier to realize experimentally and share attractive properties of antiferromagnets such as low stray magnetic fields and the absence of skyrmion Hall effect [63]. Also, magnetic textures in synthetic antiferromagnets can be driven at velocities higher than that in ferromagnets $[17,42,57]$. In such heterostructures,
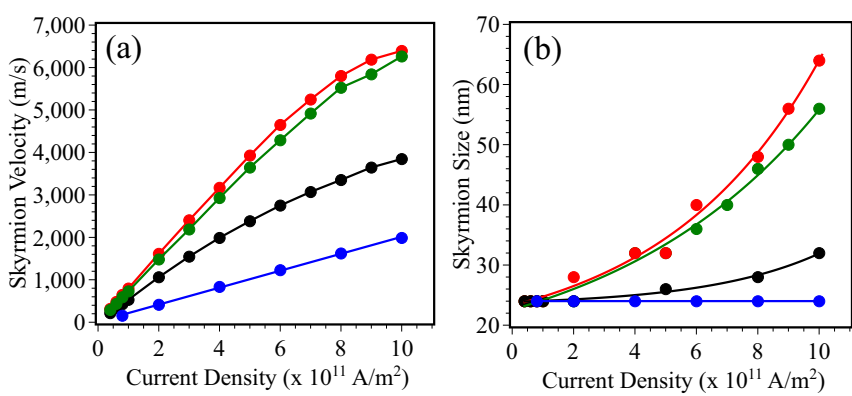

FIG. 5. Skyrmion motion in a synthetic antiferromagnet under spin Hall torque with $\theta_{\mathrm{sh}}=0.12$ (red), spin transfer torque with $\beta=200 \alpha$ (green), and topological torques, with $\lambda=1.6 \mathrm{~nm}^{2}$ (blue) and $\lambda=50 \mathrm{~nm}^{2}$ (black). In the latter two cases, we also set $\beta=\alpha$. (a) Simulated skyrmion velocity-current characteristics and (b) size variation as a function of applied current density. In (b), the solid lines are guides for the eye.

the antiferromagnetic exchange is given by Ruderman-KittelKasuya-Yosida (RKKY) interlayer exchange coupling and is therefore orders of magnitude smaller than the inter-sublattice exchange usually encountered in bulk antiferromagnets. The direct consequence is that the spin wave velocity is substantially reduced and, therefore, the nonlinear effects discussed in the previous section should be present at much lower current densities. In the simulations, we have considered two ferromagnetic layers of dimensions $1000 \times 100 \times 1 \mathrm{~nm}^{3}$, separated by a spacer layer of $1 \mathrm{~nm}$ [57]. The material parameters we use are $[64,65] M_{s}=600 \mathrm{kA} / \mathrm{m}, A_{\mathrm{FM}}=20 \mathrm{pJ} / \mathrm{m}$, $K_{z}=0.5 \mathrm{MJ} / \mathrm{m}^{3}, D=2.5 \mathrm{MJ} / \mathrm{m}^{2}$, and $\alpha=0.005$. We also set an antiferromagnetic RKKY interaction of $-10 \mathrm{MJ} / \mathrm{m}^{2}$. For these parameters, a relaxed skyrmion size of $24 \mathrm{~nm}$ is obtained. For the spin Hall torque, a spin Hall angle of $\theta_{\mathrm{sh}}=0.12$ was taken. For a comprehensive study of the influence of the materials' parameters on the properties of the synthetic antiferromagnetic skyrmion, we refer the reader to Ref. [57].

Figure 5(a) compares the simulated skyrmion current velocity curves for spin Hall torque, spin transfer torque, and topological torque. First, let us consider the skyrmion motion under spin Hall torque. Compared with bulk antiferromagnets (Fig. 2), we see that the skyrmion velocity in a synthetic antiferromagnet saturates at high current densities due to the magnetization tilting caused by the spin Hall torque. Also, a reasonably weak antiferromagnetic coupling in synthetic antiferromagnets results in a pronounced lateral expansion of the skyrmion, as seen in Fig. 5(b). Overall, while the features are qualitatively the same as in the bulk antiferromagnets, the much weaker antiferromagnetic exchange results in enhanced skyrmion deformation at much lower current density. The influence of the topological torque is also reported and results in lower skyrmion velocities but also smaller skyrmion expansion compared to the spin Hall torque. We arrive at the same conclusion as in Sec. II B: the topological torque is advantageous compared to the spin Hall torque as it results in a much smaller lateral expansion and therefore a more stable antiferromagnetic skyrmion, while allowing for reasonably high velocities to be achieved. 


\section{CONCLUSION}

In summary, we have investigated the role of selected current-driven torques on antiferromagnetic skyrmion motion through both simulations and analytical formalism. Although the skyrmions can be driven at high velocities by either spin Hall torque or topological torque, their deformation becomes quite substantial close to the velocity saturation $v_{m}$. This lateral expansion can become an important issue while designing the antiferromagnetic racetrack: as soon as the skyrmion reaches the racetrack boundaries, it transforms into two longitudinal domain walls that can lead to the loss of information [46]. A possible route to solve this hurdle is to exploit the topological torque that arises from electrons flowing inside the antiferromagnetic layer, rather than the more conventional spin Hall torque. As a matter of fact, while the current-velocity relation given by the spin Hall torque remains mostly linear in and only saturates close to the maximum velocity $v_{m}$, the one obtained with the topological torque displays a sublinear behavior at much lower velocity $v_{m} / 8$ due to its high sensitivity to the lateral skyrmion expansion $\Delta_{y}$. This saturation also means that the lateral expansion remains moderate for reasonable current densities. It therefore appears that one needs to make a compromise between skyrmion velocity and lateral expansion in the case of antiferromagnets. This aspect is exemplified in the case of synthetic antiferromagnetic skyrmions, where the much weaker exchange results in much more dramatic deformations at lower current densities. In order to make the topological torque active, one needs to ensure that most of the injected current flows inside the antiferromagnetic layer, rather than in the adjacent layers. This can be achieved quite easily in bulk noncentrosymmetric antiferromagnets, but remains challenging to realize in ultrathin magnetic multilayers. Nonetheless, such an achievement would lead to substantial benefit for antiferromagnetic skyrmion integration in applied devices.

\section{ACKNOWLEDGMENTS}

The authors acknowledge fruitful discussions with A. Abbout, C. A. Akosa, J.-V. Kim, and A. Thiaville. A.S., F.Z., and A.M. were supported by the King Abdullah University of Science and Technology (KAUST). R.T. and G.F. acknowledge the project ThunderSKY, funded by the Hellenic Foundation for Research and Innovation (HFRI) and the General Secretariat for Research and Technology (GSRT), under Grant Agreement No. 871.

\section{APPENDIX A: NONADIABATICITY PARAMETER IN ANTIFERROMAGNETS}

In ferromagnetic textures, the nonadiabatic coefficient $\beta$ is associated with the misalignment of the itinerant electron spin from the local magnetization direction, a mechanism called spin mistracking. In the limit of abrupt magnetic textures, whose characteristic length is comparable to the spin dephasing or decoherence length, this mistracking is associated with the spin-dependent reflection of the carrier against the texture [66-68]. In the opposite limit, when the magnetic texture is smooth and drift-diffusion theory applies, the nonadiabaticity is associated with spin relaxation: the incoming spin current is not entirely transferred to the local magnetization, resulting in the nonadiabatic torque contribution [53].

In a nutshell, the drift-diffusion equation governing the spin transport in a ferromagnetic texture reads $[53,68]$

$$
-\frac{1}{\tau_{\Delta}} \mathbf{m} \times \mathbf{s}+\frac{1}{\tau_{\varphi}} \mathbf{m} \times(\mathbf{s} \times \mathbf{m})+\frac{1}{\tau_{\mathrm{sf}}} \mathbf{s}=-b_{j} \partial_{x} \mathbf{m} .
$$

Here, $\tau_{\Delta}$ and $\tau_{\text {sf }}$ are the (Larmor) spin precession and spin relaxation times, while $\tau_{\varphi}$ is the spin dephasing time, ignored in Ref. [53] and accounting for the progressive alignment of the spin accumulation $\mathbf{s}$ along the magnetization $\mathbf{m} . b_{j}$ is the spin drift velocity defined in Eq. (2). The torque is therefore

$$
\begin{aligned}
\boldsymbol{\tau} & =-\frac{1}{\tau_{\Delta}} \mathbf{m} \times \mathbf{s}+\frac{1}{\tau_{\varphi}} \mathbf{m} \times(\mathbf{s} \times \mathbf{m}), \\
& =-\frac{1+\chi \xi}{1+\xi^{2}} b_{j} \partial_{x} \mathbf{m}+\frac{\tau_{\Delta}}{\tau_{\mathrm{sf}}} \frac{1}{1+\xi^{2}} b_{j} \mathbf{m} \times \partial_{x} \mathbf{m} .
\end{aligned}
$$

Here, $\chi=\tau_{\Delta} / \tau_{\varphi}$ and $\xi=\left(\tau_{\Delta} / \tau_{\varphi}+\tau_{\Delta} / \tau_{\mathrm{sf}}\right)$. In the limit $\tau_{\varphi} \rightarrow 0$, we retrieve the result of Ref. [53].

Let us now turn our attention toward collinear antiferromagnetic textures. Based on Ref. [3], the drift-diffusion equations for the coupled dynamics on the two magnetic sublattices, 1 and 2, read

$$
\frac{1}{2 \tau_{\Gamma}} \Delta \mathbf{s}-\frac{1}{\tau_{\Delta}} \mathbf{n} \times \mathbf{s}_{1}+\frac{1}{\tau_{\varphi}} \mathbf{n} \times\left(\mathbf{s}_{1} \times \mathbf{n}\right)+\frac{1}{\tau_{\mathrm{sf}}} \mathbf{s}_{1}=-b_{j} \partial_{x} \mathbf{n}
$$

$$
-\frac{1}{2 \tau_{\Gamma}} \Delta \mathbf{s}+\frac{1}{\tau_{\Delta}} \mathbf{n} \times \mathbf{s}_{2}+\frac{1}{\tau_{\varphi}} \mathbf{n} \times\left(\mathbf{s}_{2} \times \mathbf{n}\right)+\frac{1}{\tau_{\mathrm{sf}}} \mathbf{s}_{2}=b_{j} \partial_{x} \mathbf{n} .
$$

Here $\Delta \mathbf{s}=\mathbf{s}_{1}-\mathbf{s}_{2}$ and $\tau_{\Gamma}$ is the lifetime of the spin carrier on one sublattice. These equations are defined in terms of the Néel order $\mathbf{n}$, rather than the magnetization $\mathbf{m}$, but by setting $\mathbf{n}=\mathbf{m}_{1}=-\mathbf{m}_{2}$, one retrieves the equations of two coupled magnetic sublattices. In sublattice $i=1,2$, the torque reads

$$
\boldsymbol{\tau}_{i}=-\frac{1}{\tau_{\Delta}} \mathbf{m}_{i} \times \mathbf{s}_{i}+\frac{1}{\tau_{\varphi}} \mathbf{m}_{i} \times\left(\mathbf{s}_{i} \times \mathbf{m}_{i}\right) .
$$

In order to obtain a compact formula, we set $\tau_{\varphi} \gg \tau_{\Delta}, \tau_{\mathrm{sf}}, \tau_{\Gamma}$. As a matter of fact, the spin dephasing process is much slower in antiferromagnets than in ferromagnets. In ferromagnets, it arises from the interference of continuously precessing itinerant spins around the local magnetization [69]. In the diffusive limit, this dephasing time reads $\tau_{\varphi}^{\mathrm{F}} \sim \tau_{\Delta}^{2} / \tau$ (see, e.g., Ref. [70]). In antiferromagnets, the spin precession around the magnetic moment of one sublattice is limited by the lifetime $\tau_{\Gamma}$. Therefore, the dephasing reads $\tau_{\varphi}^{\mathrm{AF}} \sim \tau_{\Delta}^{2} / \tau_{\Gamma}$. Since the time between two momentum scattering events is much larger than the lifetime of the spin carrier on a given sublattice, $\tau \gg \tau_{\Gamma}$, and one expects the spin dephasing to be much longer in antiferromagnets than in ferromagnets, $\tau_{\varphi}^{\mathrm{AF}} \gg \tau_{\varphi}^{\mathrm{F}}$.

We therefore neglect the spin dephasing in Eqs. (A4)-(A6). After straightforward algebra, we obtain the expression of the torque on sublattice $i$,

$$
\boldsymbol{\tau}_{i}=\mp \frac{1}{1+v^{2}} b_{j} \partial_{x} \mathbf{n}+\frac{\tau_{\Delta}}{\tau_{\mathrm{sf}}} \frac{1}{1+v^{2}} b_{j} \mathbf{n} \times \partial_{x} \mathbf{n},
$$



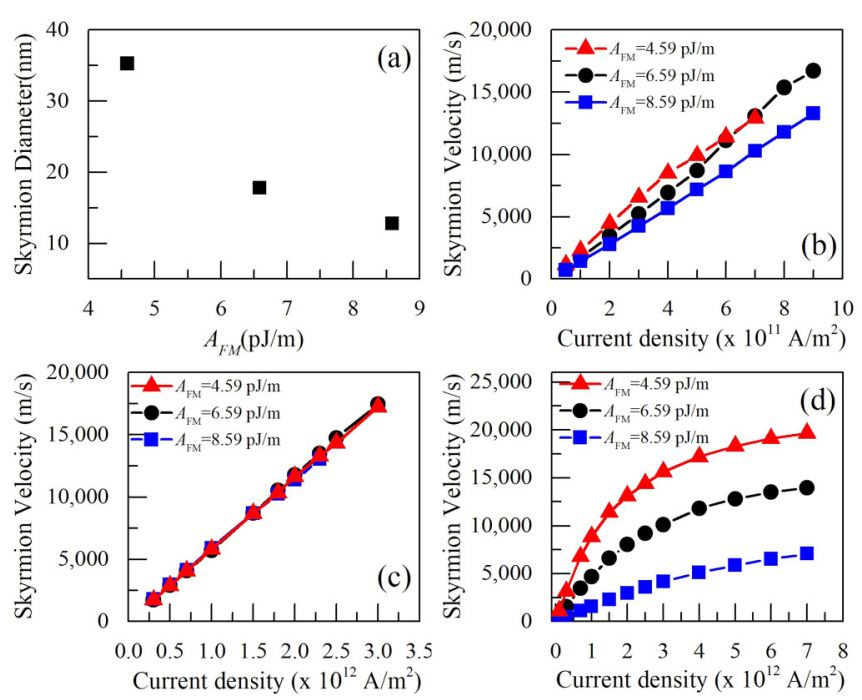

FIG. 6. (a) Skyrmion diameter as a function of $A_{\mathrm{FM}}$, for $A_{\mathrm{AFM}}=$ $-6.59 \mathrm{pJ} / \mathrm{m}$. Skyrmion velocity as a function of the current density when driven by the (b) spin Hall torque, (c) nonadiabatic torque with $\beta=100 \alpha$, and (d) topological torque with $\lambda^{2}=13 \mathrm{~nm}^{2}$, for three values of $A_{\mathrm{FM}}$ and $A_{\mathrm{AFM}}=-6.59 \mathrm{pJ} / \mathrm{m}$.

where $+(-)$ corresponds to sublattice $1(2)$, and $v^{2}=$ $\tau_{\Delta}^{2} / \tau_{\Gamma} \tau_{\text {sf }}$. This expression demonstrates that the nonadiabaticity parameter $\beta$ in diffusive antiferromagnets has the same origin as in ferromagnets, in spite of the much longer spin dephasing time.

\section{APPENDIX B: PARAMETER DEPENDENCE OF SKYRMION PROPERTIES}

In order to provide a comprehensive description of the antiferromagnetic skyrmion motion, we report further micromagnetic simulations for different sets of parameters. Figure 6 shows the skyrmion velocities when the intrasublattice ferromagnetic constant $A_{\mathrm{FM}}$ changes. Statically, tuning $A_{\mathrm{FM}}$ changes the equilibrium skyrmion size $\Delta_{0}$. We observe in Fig. 6(a) that the skyrmion diameter decreases as $A_{\mathrm{FM}}$ increases. This behavior has a direct consequence on the skyrmion velocities, similarly to what happens in ferromagnets [71]. In particular, for the case of the spin Hall torque, the analytical velocity is proportional to the $x$-lateral dimension of the skyrmion $v_{\mathrm{sh}} \propto \tau_{\mathrm{sh}} \Delta_{x}$ [see Eq. (19)]; therefore smaller skyrmions move slower than larger skyrmions. This is consistent with the micromagnetic results of Fig. 6. Figure 6(b) shows the skyrmion velocity as a function of the current density under the spin Hall torque. One can see that the smaller skyrmion for $A_{\mathrm{FM}}=8.59 \mathrm{pJ} / \mathrm{m}$ exhibits a smaller velocity than the larger skyrmion for $A_{\mathrm{FM}}=4.59 \mathrm{pJ} / \mathrm{m}$ and no sublinear behavior is observed. On the other hand, the skyrmion velocity for $A_{\mathrm{FM}}=4.59 \mathrm{pJ} / \mathrm{m}$ undergoes a sublinear trend at very small current densities $\left(\sim 2 \times 10^{11} \mathrm{~A} / \mathrm{m}^{2}\right)$ where the skyrmion rapidly expands, but at $j_{e}=6 \times 10^{11} \mathrm{~A} / \mathrm{m}^{2}$ the velocity becomes the same as the one for $A_{\mathrm{FM}}=6.59$ $\mathrm{pJ} / \mathrm{m}$. This is because the skyrmion expansion is limited by the confining potential originating from the sample boundaries. Hence, if the size approaches the same value, the
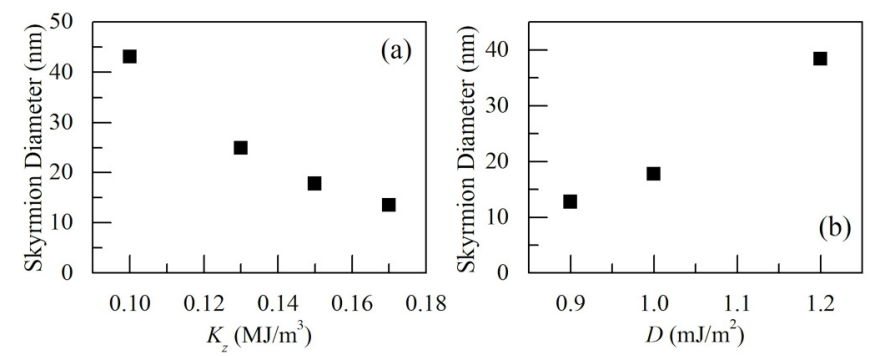

FIG. 7. Skyrmion diameter as a function of the (a) perpendicular anisotropy constant $K_{z}$, and (b) DMI parameter $D$, for $A_{\mathrm{FM}}=$ $-A_{\mathrm{AFM}}=6.59 \mathrm{pJ} / \mathrm{m}$.

velocity is also the same. Moreover, for current larger than $7 \times 10^{11} \mathrm{~A} / \mathrm{m}^{2}$, the expanding skyrmion reaches the sample edges and it is then converted into two domain walls, thus preventing us from applying larger currents.

The $A_{\mathrm{FM}}$ constant does not affect the skyrmion velocity in the nonadiabatic torque case [Fig. 6(c)], as also expected from the analytical expression $v=\beta b_{j} / \alpha$ [Eq. (20)], where the velocity solely depends on the ratio $\beta / \alpha$. However, the $A_{\mathrm{FM}}$ affects again the maximum applicable current, which is $2.3 \times 10^{12} \mathrm{~A} / \mathrm{m}^{2}$ for $A_{\mathrm{FM}}=4.59 \mathrm{pJ} / \mathrm{m}$. When turning on the topological torque, the analytical velocity due to such a torque depends on the skyrmion lateral size $v \propto 1 / \Delta_{y}^{2}$ [see Eq. (22)]; therefore smaller skyrmions undergo a larger torque and hence a faster motion. Micromagnetic simulations reported in Fig. 6(d) confirm this behavior.

Furthermore, we wish to stress that the other parameters that influence the equilibrium size of the skyrmion are the DMI and the perpendicular anisotropy (Fig. 7). In particular, a larger DMI (or smaller perpendicular anisotropy $K_{z}$ ) promotes the formation of larger skyrmions and vice versa.
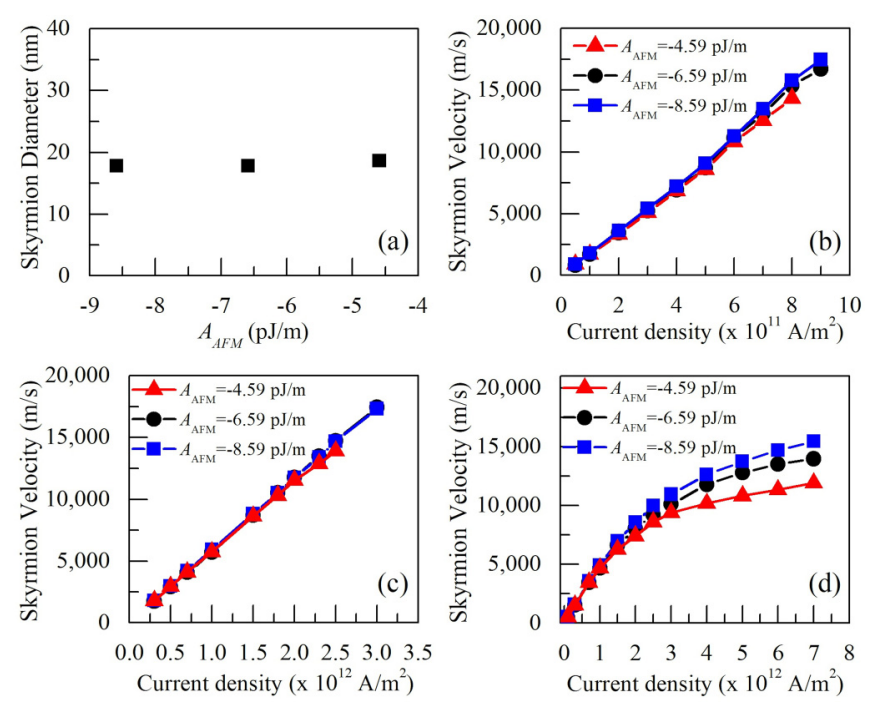

FIG. 8. (a) Skyrmion diameter as a function of $A_{\mathrm{AFM}}$, when $A_{\mathrm{FM}}$ is fixed to $6.59 \mathrm{pJ} / \mathrm{m}$. Skyrmion velocity as a function of the (b) spin Hall torque current density, (c) nonadiabatic torque current density when $\beta=100 \alpha$, (d) including the topological torque when $\lambda^{2}=$ $13 \mathrm{~nm}^{2}$, for three values of $A_{\mathrm{AFM}}$ and $A_{\mathrm{FM}}=6.59 \mathrm{pJ} / \mathrm{m}$. 

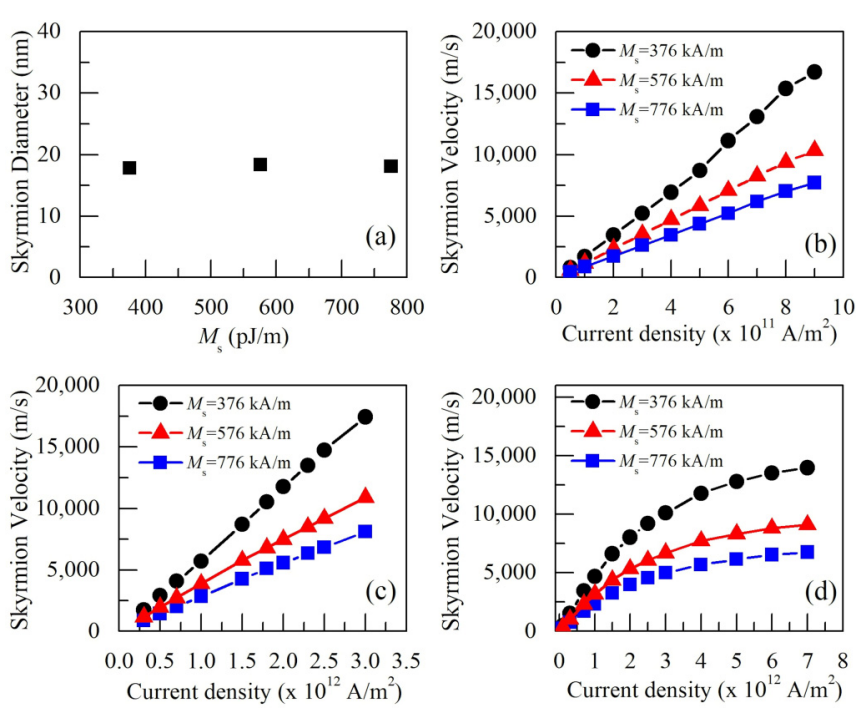

FIG. 9. (a) Skyrmion diameter as a function of $M_{\mathrm{s}}$, when $A_{\mathrm{FM}}=$ $-A_{\mathrm{AFM}}=6.59 \mathrm{pJ} / \mathrm{m}$. Skyrmion velocity as a function of the current density when driven by the (b) spin Hall torque, (c) nonadiabatic torque with $\beta=100 \alpha$, and (d) topological torque with $\lambda^{2}=13 \mathrm{~nm}^{2}$, for three values of $M_{\mathrm{s}}$ and $A_{\mathrm{FM}}=-A_{\mathrm{AFM}}=6.59 \mathrm{pJ} / \mathrm{m}$.

Figure 8 displays the skyrmion properties when changing the inter-sublattice antiferromagnetic exchange constant $A_{\mathrm{AFM}}$. In this case, the skyrmion size does not change with the $A_{\mathrm{AFM}}$. Therefore, similar velocity dependencies on $A_{\mathrm{AFM}}$ are obtained for the spin Hall torque [Fig. 8(a)] and nonadiabatic torque [Fig. 8(b)]. When the skyrmion is driven by the topological torque, the analytical velocity $v=v_{\text {top }} v_{m} /\left(v_{\text {top }}-\right.$ $\left.v_{m} / 8\right)$ [Eq. (22)] depends on $v_{m}=\sqrt{\left(A_{\mathrm{AFM}}+\Delta_{0} C_{2} D / 4\right) / \rho}$. Therefore, larger velocities are obtained for larger magnitude of $A_{\mathrm{AFM}}$, as obtained also in the micromagnetic simulations [see Fig. 8(d)].

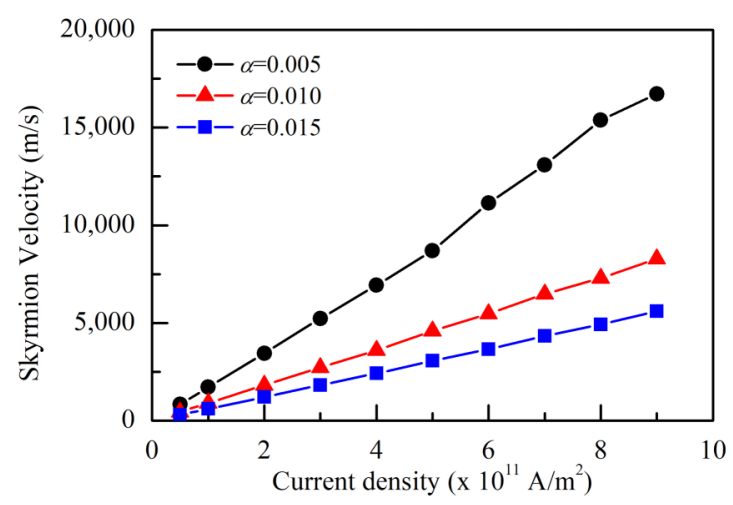

FIG. 10. Skyrmion velocity as a function of the current density when driven by the spin Hall torque for three values of $\alpha$, with $A_{\mathrm{FM}}=$ $-A_{\mathrm{AFM}}=6.59 \mathrm{pJ} / \mathrm{m}$ and $M_{\mathrm{s}}=376 \mathrm{kA} / \mathrm{m}$.

Figure 9 shows the effect of the change of the saturation magnetization $M_{\mathrm{s}}$. For the range of $M_{\mathrm{s}}$ analyzed here, the equilibrium skyrmion diameter does not significantly change [Fig. 9(d)]. On the other hand, the skyrmion velocity decreases as a function of $M_{\mathrm{s}}$ for a fixed current density, as expected from the torque coefficient which is proportional to $1 / M_{\mathrm{s}}$ for all three cases: spin Hall torque [Fig. 9(b)], nonadiabatic torque [Fig. 9(c)], and topological torque [Fig. 9(d)].

Figure 10 shows the skyrmion velocity as a function of the damping constant $\alpha$. We only show the spin Hall torque case because the effect of the $\beta / \alpha$ ratio on the skyrmion velocity driven by the nonadiabatic torque has already been displayed in Figs. 3 and 4. As expected, the velocity decreases upon increasing the damping as confirmed analytically, $v_{\text {sh }} \propto 1 / \alpha$ [see Eq. (19)].
[1] V. Baltz, A. Manchon, M. Tsoi, T. Moriyama, T. Ono, and Y. Tserkovnyak, Rev. Mod. Phys. 90, 015005 (2018).

[2] J. Železný, H. Gao, K. Výborný, J. Zemen, J. Mašek, A. Manchon, J. Wunderlich, J. Sinova, and T. Jungwirth, Phys. Rev. Lett. 113, 157201 (2014).

[3] A. Manchon, J. Phys. Condens. Matter 29, 104002 (2017).

[4] P. Wadley, B. Howells, J. Elezny, C. Andrews, V. Hills, R. P. Campion, V. Novak, K. Olejnik, F. Maccherozzi, S. S. Dhesi, S. Y. Martin, T. Wagner, J. Wunderlich, F. Freimuth, Y. Mokrousov, J. Kune, J. S. Chauhan, M. J. Grzybowski, A. W. Rushforth, K. W. Edmonds, B. L. Gallagher, and T. Jungwirth, Science 351, 587 (2016).

[5] X. Z. Chen, R. Zarzuela, J. Zhang, C. Song, X. F. Zhou, G. Y. Shi, F. Li, H. A. Zhou, W. J. Jiang, F. Pan, and Y. Tserkovnyak, Phys. Rev. Lett. 120, 207204 (2018).

[6] T. Moriyama, K. Oda, T. Ohkochi, M. Kimata, and T. Ono, Sci. Rep. 8, 14167 (2018).

[7] T. Moriyama, W. Zhou, T. Seki, K. Takanashi, and T. Ono, Phys. Rev. Lett. 121, 167202 (2018).

[8] T. Jungwirth, X. Marti, P. Wadley, and J. Wunderlich, Nat. Nanotechnology 11, 231 (2016).
[9] T. Jungwirth, J. Sinova, A. Manchon, X. Marti, J. Wunderlich, and C. Felser, Nat. Phys. 14, 200 (2018).

[10] S. S. P. Parkin, M. Hayashi, and L. Thomas, Science (New York, N.Y.) 320, 190 (2008).

[11] J. Slonczewski, J. Magn. Magn. Mater. 159, L1 (1996).

[12] L. Berger, Phys. Rev. B 54, 9353 (1996).

[13] A. Manchon, J. Železný, I. M. Miron, T. Jungwirth, J. Sinova, A. Thiaville, K. Garello, and P. Gambardella, Rev. Mod. Phys. 91, 035004 (2019).

[14] K. M. D. Hals, Y. Tserkovnyak, and A. Brataas, Phys. Rev. Lett. 106, 107206 (2011).

[15] E. Tveten, A. Qaiumzadeh, O. Tretiakov, and A. Brataas, Phys. Rev. Lett. 110, 127208 (2013).

[16] O. Gomonay, T. Jungwirth, and J. Sinova, Phys. Rev. Lett. 117, 017202 (2016).

[17] T. Shiino, S.-h. Oh, P. M. Haney, S.-w. Lee, G. Go, B.-g. Park, and K.-j. Lee, Phys. Rev. Lett. 117, 087203 (2016).

[18] N. Nagaosa and Y. Tokura, Nat. Nanotechnol. 8, 899 (2013).

[19] A. Fert, V. Cros, and J. Sampaio, Nat. Nanotechnol. 8, 152 (2013). 
[20] A. Bogdanov and U. Rößler, Phys. Rev. Lett. 87, 037203 (2001).

[21] U. K. Roessler, A. N. Bogdanov, and C. Pfleiderer, Nature (London) 442, 797 (2006).

[22] S. Mühlbauer, B. Binz, F. Jonietz, C. Pfleiderer, A. Rosch, A. Neubauer, R. Georgii, and P. Böni, Science (New York, N.Y.) 323, 915 (2009).

[23] X. Z. Yu, Y. Onose, N. Kanazawa, J. H. Park, J. H. Han, Y. Matsui, N. Nagaosa, and Y. Tokura, Nature (London) 465, 901 (2010).

[24] Z. G. Yu, Phys. Rev. Lett. 106, 106602 (2011).

[25] S. Heinze, K. von Bergmann, M. Menzel, J. Brede, A. Kubetzka, R. Wiesendanger, G. Bihlmayer, and S. Blügel, Nat. Phys. 7, 713 (2011).

[26] N. Romming, C. Hanneken, M. Menzel, J. E. Bickel, B. Wolter, K. von Bergmann, A. Kubetzka, and R. Wiesendanger, Science (New York, N.Y.) 341, 636 (2013).

[27] B. Dupé, M. Hoffmann, C. Paillard, and S. Heinze, Nat. Commun. 5, 4030 (2014).

[28] B. Dupé, G. Bihlmayer, S. Blügel, and S. Heinze, Nat. Commun. 7, 11779 (2016).

[29] T. Schulz, R. Ritz, A. Bauer, M. Halder, M. Wagner, C. Franz, C. Pfleiderer, K. Everschor, M. Garst, and A. Rosch, Nat. Phys. 8, 301 (2012).

[30] J. Sampaio, V. Cros, S. Rohart, A. Thiaville, and A. Fert, Nat. Nanotechnol. 8, 839 (2013).

[31] J. Iwasaki, M. Mochizuki, and N. Nagaosa, Nat. Commun. 4, 1463 (2013).

[32] J. Iwasaki, M. Mochizuki, and N. Nagaosa, Nat. Nanotechnol. 8, 742 (2013).

[33] C. Reichhardt, D. Ray, and C. J. Olson Reichhardt, Phys. Rev. Lett. 114, 217202 (2015).

[34] C. Reichhardt and C. Olson Reichhardt, New J. Phys. 18, 095005 (2016).

[35] D. Maccariello, W. Legrand, N. Reyren, K. Garcia, K. Bouzehouane, S. Collin, V. Cros, and A. Fert, Nat. Nanotechnol. 13, 233 (2018).

[36] A. Salimath, A. Abbout, A. Brataas, and A. Manchon, Phys. Rev. B 99, 104416 (2019).

[37] A. Abbout, J. Weston, X. Waintal, and A. Manchon, Phys. Rev. Lett. 121, 257203 (2018).

[38] A. N. Bogdanov, U. K. Roessler, M. Wolf, and K. H. Muller, Phys. Rev. B 66, 214410 (2002).

[39] B. A. Ivanov and A. K. Kolezhuk, Low Temp. Phys. (Fiz. Nizk. Temp.) 21, 355 (1995).

[40] X. Zhang, M. Ezawa, and Y. Zhou, Sci. Rep. 5, 9400 (2015).

[41] J. Barker and O. A. Tretiakov, Phys. Rev. Lett. 116, 147203 (2016).

[42] X. Zhang, Y. Zhou, and M. Ezawa, Nat. Commun. 7, 10293 (2016).

[43] B. Göbel, A. Mook, J. Henk, and I. Mertig, Phys. Rev. B 96, 060406(R) (2017).

[44] P. M. Buhl, F. Freimuth, S. Blügel, and Y. Mokrousov, Phys. Status Solidi RRL 4, 1700007 (2017).

[45] C. A. Akosa, O. A. Tretiakov, G. Tatara, and A. Manchon, Phys. Rev. Lett. 121, 097204 (2018).
[46] C. Jin, C. Song, J. Wang, Q. Liu, C. Jin, C. Song, J. Wang, and Q. Liu, Appl. Phys. Lett. 109, 182404 (2016).

[47] F. D. M. Haldane, Phys. Rev. Lett. 50, 1153 (1983).

[48] A. M. Kosevich, in Solitons, edited by S. Trullinger, V. Zakharov, and V. Pokrovsky (Elsevier, Amsterdam, 1986), Chap. 11, p. 555.

[49] S. K. Kim, Y. Tserkovnyak, and O. Tchernyshyov, Phys. Rev. B 90, 104406 (2014).

[50] M. E. Lucassen, H. J. van Driel, C. M. Smith, and R. A. Duine, Phys. Rev. B 79, 224411 (2009).

[51] B. A. Ivanov, Low Temp. Phys. 40, 91 (2014).

[52] J. Kim, J. Sinha, S. Mitani, M. Hayashi, S. Takahashi, S. Maekawa, M. Yamanouchi, and H. Ohno, Phys. Rev. B 89, 174424 (2014).

[53] S. Zhang and Z. Li, Phys. Rev. Lett. 93, 127204 (2004).

[54] V. P. Kravchuk, J. Magn. Magn. Mater. 367, 9 (2014).

[55] R. Tomasello, E. Martinez, R. Zivieri, L. Torres, M. Carpentieri, and G. Finocchio, Sci. Rep. 4, 6784 (2014).

[56] V. Puliafito, R. Khymyn, M. Carpentieri, B. Azzerboni, V. Tiberkevich, A. Slavin, and G. Finocchio, Phys. Rev. B 99, 024405 (2019).

[57] R. Tomasello, V. Puliafito, E. Martinez, A. Manchon, M. Ricci, M. Carpentieri, and G. Finocchio, J. Phys. D 50, 325302 (2017).

[58] A. Bisig, C. A. Akosa, J.-H. Moon, J. Rhensius, C. Moutafis, A. von Bieren, J. Heidler, G. Kiliani, M. Kammerer, M. Curcic, M. Weigand, T. Tyliszczak, B. Van Waeyenberge, H. Stoll, G. Schütz, K.-J. Lee, A. Manchon, and M. Kläui, Phys. Rev. Lett. 117, 277203 (2016).

[59] C. A. Akosa, P. B. Ndiaye, and A. Manchon, Phys. Rev. B 95, 054434 (2017).

[60] M. Hayashi, L. Thomas, Y. B. Bazaliy, C. Rettner, R. Moriya, X. Jiang, and S. S. P. Parkin, Phys. Rev. Lett. 96, 197207 (2006).

[61] M. Eltschka, M. Wötzel, J. Rhensius, S. Krzyk, U. Nowak, M. Kläui, T. Kasama, R. E. Dunin-Borkowski, L. J. Heyderman, H. J. van Driel, and R. A. Duine, Phys. Rev. Lett. 105, 056601 (2010).

[62] C. Burrowes, A. P. Mihai, D. Ravelosona, J.-V. Kim, C. Chappert, L. Vila, A. Marty, Y. Samson, F. Garcia-Sanchez, L. D. Buda-Prejbeanu, I. Tudosa, E. E. Fullerton, and J.-P. Attane, Nat. Phys. 6, 17 (2010).

[63] R. A. Duine, K.-J. Lee, S. S. P. Parkin, and M. D. Stiles, Nat. Phys. 14, 217 (2018).

[64] K.-S. Ryu, S.-H. Yang, L. Thomas, and S. S. P. Parkin, Nat. Commun. 5, 3910 (2014).

[65] S.-H. Yang, K.-S. Ryu, and S. Parkin, Nat. Nanotechnol. 10, 221 (2015).

[66] G. Tatara and H. Kohno, Phys. Rev. Lett. 92, 086601 (2004).

[67] J. Xiao, A. Zangwill, and M. Stiles, Phys. Rev. B 73, 054428 (2006).

[68] C. A. Akosa, W.-S. Kim, A. Bisig, M. Kläui, K.-J. Lee, and A. Manchon, Phys. Rev. B 91, 094411 (2015).

[69] M. Stiles and A. Zangwill, Phys. Rev. B 66, 014407 (2002).

[70] X. Wang and A. Manchon, Phys. Rev. Lett. 108, 117201 (2012).

[71] J. Ding, X. Yang, and T. Zhu, J. Phys. D: Appl. Phys. 48, 115004 (2015). 